\title{
New Technologies
}

\section{NT1-37 Functional Genomics in Clinical Medicine Clinical implications of systems biology and high-throughput technology in pediatrics Mikael Benson \\ The Queen Silvia Children's Hospital, , Gothenburg, Sweden}

Complex diseases like diabetes, allergy and cancer depend on multiple interacting genes. It is probably not possible to find a key gene in such diseases, a "silver bullet" that can be used for diagnostic or therapeutic purposes. New technologies, like DNA microarrays allow simultaneous analysis of expression of all human genes. This may contribute to understanding of complex diseases. However, getting a functional understanding of the large datasets generated by DNA microarray studies is a significant challenge. One such study may identify hundreds or even thousands of genes that differ in expression between patients and controls. While this may be seen as discouraging it does reflect the complexity of the diseases that we study. Moreover, if we can get a grip on this complexity this may be used for clinical purposes, for example to find combinations of markers for individualized therapy. Systems biology is an emerging discipline that aims to understand how complex systems relate to biological effects. One important theoretical framework is networkbased analysis. In the context of DNA microarray studies, genes that differ in expression between patients and controls are organized in gene interaction networks. Such networks can be analyzed in a top-down fashion. The idea is the same as a microscopic examination. You start with a low magnification to identify general features of the sample and then proceed to detailed studies of individual cells. A gene interaction network can be analyzed to find sub-networks or pathways with distinct functions, such as cell proliferation or apoptosis. These sub-networks can be further dissected to find up-stream genes with putative key regulatory functions. Typically, several sub-networks or pathways are involved in complex diseases. However, a top-down analysis may result in identification of combinations of key genes that can be used for clinical purposes, such as to find markers for personalized medication. At present, there are few examples of clinical systems biological applications. However, in oncology, customized DNA microarrays are tried to find gene expression signatures for personalized medication in breast cancer. Further studies are required to define the clinical implications of systems biology and high-throughput technology in pediatrics.
NT1-38 Functional Genomics in Clinical Medicine

Proteomics in endocrine research and practice Elke Clynen; Evy Vierstraete; Geert Baggerman; Peter Verleyen; Liliane Schoofs

K.U.Leuven, Lab Developmental Physiology, Genomics, Proteomics, Leuven, Belgium

Proteomics and peptidomics represent global strategies, where all proteins or peptides present in a biological sample derived from a cell, tissue, body liquid, or whole organism are simultaneously visualised and identified. Both technologies target different windows of the proteome and therefore require a specific methodology.

Two-dimensional polyacrylamide gel electrophoresis is the key technology for proteomics. Here, proteins are separated according to their isoelectric point and molecular weight, allowing the separation of several thousand proteins in a single gel and downstream identification by mass spectrometry. For an accurate quantitative comparison of different samples with statistical confidence, 2D-DIGE (2D-difference gel electrophoresis) is the method of choice. Here, multiple protein extracts are labelled with different fluorescent dyes and subsequently separated on the same $2 \mathrm{D}$ gel. A reference sample is used, known as an internal standard, which comprises equal amounts of all biological samples in the experiment, controlling for non-biologic variation. Biologically active peptides are important substances that transmit and regulate bio-information in the circulatory and neuronal systems. This restricted window of the proteome $(<10 \mathrm{kDa})$ is however not covered by conventional gel electrophoresis and prompted the development of other extraction procedures and separation tools. This new research field was termed peptidomics. The most common tool used for peptidomics is a combination of nanoscale liquid chromatography, tandem mass spectrometry and database mining, which allows the detection and sequencing of low concentrations of peptides from complex mixtures with a high degree of automation.

Both proteomics and peptidomics are important tools in the study of the mechanism of diseases, the investigation for diagnostic biomarkers and pinpointing of novel therapeutic targets.

\section{NT2-39 Refinement of Measurements \\ Gas chromatography-mass spectrometry profiling of steroids in times of molecular biology \\ Stefan A. Wudy \\ Justus Liebig University, Pediatric Endocrinology/Diabetology, Giessen, Germany}

The aim of this presentation is to outline the potential of gas chromatographymass spectrometry profiling of steroids in the diagnosis of endogenous human steroid disorders. Mass spectrometry currently provides the highest specificity in steroid analysis. The non-invasive and non-selective GC-MS urinary steroid profiling technique enables diagnosis of most adrenal enzyme defects in steroid biosynthesis. While enzymatic defects can be diagnosed from spot urine samples in most cases, analysis of 24-hr urinary samples permits determination of hormonal excretion rates or enables diagnostic or therapeutic monitoring of steroid related diseases. Profiling plasma steroids by isotope dilution/GC-MS is particularly suitable where only minimal plasma samples are available and/or the highest specificity is required. In conclusion, GCMS steroid profiling presents a complementary analytical technique whenever highest specificity is required. Clinical GC-MS profiling of steroids is also highly recommended as a reasonable initial diagnostic approach - especially in unclear situations - avoiding uncritical and expensive attempts at molecular diagnostic testing. 


\section{NT2-40 Refinement of Measurements}

\section{Steroid metabolomics using GC-MS}

Carlos Knopf'; Oshrat Baruch'; Eran Eden²; Zohar Yakhini2; Ze'ev Hochberg ${ }^{3}$

${ }^{1}$ Rambam Medical Center, Dep of Pediatric Endocrinology \& Clinical Biochem., Haifa, Israel; ${ }^{2}$ Rambam Medical Center \& Technion Faculty of Med., Dep of Pediatric Endocrinology \& Clinical Biochem., Haifa, Israel; 3Israel Technion Institute of Technology, Faculty of Computer Science, Haifa, Israel

Metabolomics is the study of complex metabolite profiles in biological samples, focusing on all, rather than some, of the metabolites in a given sample. Of particular interest to Metabolomics are small, low-molecular-weight compounds that serve as substrates and products in metabolic pathways. These include fatty acids (medium, long, very long, branched chain), amino acids, organic acids and steroids, all of which are measured in our Laboratory. The steroids metabolome is the set of all of the steroids and their metabolites in a given individual, and it is likely that every one has his/her own unique fingerprint. Every adrenal or gonadal disease has its characteristic pattern of steroid metabolites. Complex steroid-related disorders (CSRD), like obesity, insulin resistance, hirsutism, PCOS, baldness, prostate hypertrophy and hypertension, might have characteristic steroid Metabolomics which could be defined through bioinformatic technology. GC-MS Profiling of Steroids generates a large amount of information, difficult to be analyzed manually. Computerized programs can analyze data at a rate and level of complexity that is impossible to do by hand. Moreover, these programs are able to find subtle relations which the researcher can not uncover with his eyes. Such high throughput data require the application of automated and algorithmic approaches, like data mining, to identify trends or anomalies, seeking for relationships that have not previously been discovered. A program was designed for searching metabolites and metabolite quotients, each representing unique enzymatic activity, that best differentiate between members of two particular groups. The software identifies significant metabolites for both known diseases and CSRD, without knowledge of the meaning of the data or the disease mechanism. The potential of linking analytical Metabolomics with bioinformatics data is still in its early life, and will probably reveal unexpected properties and associations of biological systems. 